\title{
Influência da Capacidade de Rede e do Compartilhamento de Informações e de Conhecimento na Inovação Aberta
}

\author{
Silvana Mannes ${ }^{(D)}$, llse Maria Beuren ${ }^{(D)}$ \\ Universidade Federal de Santa Catarina. Florianópolis-SC. Brazil.
}

$凶$

'silvanamannes@yahoo.com.br

2ilse.beuren@gmail.com
Editado por:

Orleans Silva Martins

Paulo Roberto da Cunha

\section{Resumo}

Objetivo: Este artigo analisa a influência da capacidade de rede e do compartilhamento de informações e de conhecimento na inovação aberta em startups relacionadas com grandes empresas.

Método: Uma survey foi realizada com gestores de startups ranqueadas na plataforma 100 Open Startups. A população da pesquisa compreende 324 startups e a amostra as 144 respostas válidas. Para a análise dos dados aplicou-se a modelagem de equações estruturais pelos mínimos quadrados parciais. brasileiro e do consenso de mercado dos analistas sell-side extraídos da Bloomberg.

Resultados: Os resultados revelaram influência direta e significativa entre compartilhamento de informações e inovação aberta, assim como entre compartilhamento de conhecimento e inovação aberta. Embora não se tenha encontrado influência direta da capacidade de rede na inovação aberta, foi observada mediação do compartilhamento de informações e conhecimento nessa relação, o que indica que a capacidade de rede pode ser um importante antecedente e impactar de maneira indireta no desempenho da inovação aberta. Conclui-se que as variáveis investigadas são importantes propulsores do desempenho da inovação aberta, de modo a reduzir riscos e trazer benefícios às startups nas relações interorganizacionais

Contribuições: Os resultados contribuem com a literatura ao revelar o efeito mediador do compartilhamento de informações e conhecimento na relação entre capacidade de rede e desempenho da inovação aberta, um avanço no entendimento sobre recursos compartilhados. Também trazem implicações práticas e sociais, na medida em que fornecem informações sobre relacionamentos interorganizacionais. Espera-se que os achados auxiliem os gestores na condução do relacionamento, com o propósito de reduzir assimetrias entre os envolvidos e direcionar para uma relação benéfica de ambas as partes.

Palavras-chave: Capacidade de rede, Compartilhamento de informações, Compartilhamento de conhecimento, Inovação aberta.

How to cite:

Mannes, S., \& Beuren, I. M. (2021). INFLUÊNCIA DA CAPACIDADE DE REDE E DO COMPARTILHAMENTO DE INFORMAÇÕES E DE CONHECIMENTO NA INOVAÇÃO ABERTA. Advances in Scientific and Applied Accounting, 14(2). https://doi.org/10.14392/asaa.2021 140207 


\section{Introdução}

A Teoria da Dependência de Recursos (TDR) enfatiza o modo como as empresas lidam e se ajustam às contingências externas e a habilidade de manter e adquirir recursos que possam contribuir para sua sobrevivência (Pfeffer \& Salancik, 1978). Um pressuposto básico é que a dependência de recursos críticos direciona as ações, comportamentos e decisões das organizações (Pfeffer \& Salancik, 1978). Essa teoria fornece uma lente que permite focar na maneira pela qual se formam os relacionamentos interorganizacionais (RIOs) (Klein \& Pereira, 2016).

Um campo fértil nesta direção é o das startups, por carecerem de infraestrutura e conhecimento de mercado e terem limitações de recursos (Fabrício et al., 2015). RIOs e redes de colaboração têm sido adotados por startups para obterem mais eficiência e resultados (Klein \& Pereira, 2016) e como estratégia para assegurar sua continuidade. O envolvimento em redes ou alianças de cooperação pode proporcionar vantagem competitiva (Porter, 1990; Singh et al., 2019), mas também pode trazer desafios e comportamentos oportunistas nos relacionamentos (Parida \& Örtqvist, 2015). Para tentar reduzir a dependência, as organizações precisam gerenciar e constituir ambientes negociáveis favoráveis à empresa e estratégias eficazes para a obtenção desses recursos (Pfeffer \& Salancik, 1978).

A capacidade de rede desempenha uma função significativa no sucesso da rede (Parida et al., 2017), caracteriza-se como a capacidade de iniciar, manter e utilizar relacionamentos com parceiros externos (Walter et al., 2006). Nessa perspectiva, o presente estudo foca na análise da gestão de redes, por meio das dimensões interligadas da capacidade de rede.

Pesquisas focaram em responder questões relativas à capacidade de rede, inovação e desempenho, e encontraram resultados positivos nos efeitos da capacidade de rede na inovação e dessa no desempenho (Mitrega et al., 2017; Parida et al., 2017). Acredita-se que a capacidade de rede possa explicar o fato de que algumas empresas atingem os objetivos em relação à inovação e outras não, especialmente a inovação aberta, pois tais capacidades ajudam as empresas no gerenciamento da relação (Walter et al., 2006). A capacidade de rede permite maior vínculo, o que favorece gerenciar riscos e trazer confiança no relacionamento com parceiros (Walter et al., 2006; Parida et al., 2017).

Um dos aspectos essenciais da colaboração entre parceiros é o compartilhamento de informações e conhecimento. O compartilhamento de informações e conhecimento é considerado valioso por estreitar relações e proporcionar cooperação e colaboração na rede (Kim \& Chai, 2017). Ipe (2003) argumenta que esse compartilhamento é mais facilmente realizado quando se tem confiança e vínculo com os parceiros.

O compartilhamento de informações e conhecimento tem sido estudado em diferentes âmbitos, entre eles, na inovação aberta (Chesbrough, 2003; Gupta \& Polonsky, 2014). Pesquisas pregressas apontam influência positiva e benefícios do compartilhamento de informações e conhecimento na inovação aberta (Gupta \& Polonsky, 2014; Singh et al., 2019). Ainda, destacam a relevância do gerenciamento de relacionamentos com parceiros externos na inovação aberta (Popa et al., 2017).

A inovação aberta, como uma das práticas que inserem as organizações em redes, tradicionalmente tinha como principal estratégia a interação com universidades, centros de pesquisa e tecnologia, porém, há uma tendência crescente de promover relacionamentos entre grandes empresas e startups (Chesbrough, 2003; Stal et al., 2014). A literatura revela que o foco da inovação aberta prevalece em grandes empresas, mas dados referentes a inovação aberta de startups, principalmente em âmbito nacional, ainda são incipientes (Popa et al., 2017; Cajuela \& Galina, 2020).

As pequenas empresas podem ganhar com a inovação aberta, visto que já possuem esforços voltados para fora da organização devido aos seus mercados e recursos limitados (Lee et al., 2010). Porém, devido à limitação de recursos, empresas menores possuem dificuldades de manter e desenvolver RIOs colaborativos (Fabrício et al., 2015). No caso das startups, de um lado carecem de espaço no mercado, infraestrutura e experiência, de outro lado possuem flexibilidade e agilidade e são constituídas de boas ideias e invenções (Fabrício et al., 2015).

Isso atrai grandes empresas e parceiros para RIOs. Por isso, pesquisas sobre inovação aberta em pequenas empresas vêm obtendo interesse crescente (Radziwon \& Bogers, 2019). No entanto, ainda falta uma compreensão quanto ao gerenciamento e às condições em que empresas menores podem ter êxito na inovação aberta (Radziwon \& Bogers, 2019).

Frente aos apontamentos reportados, o objetivo do 
estudo é analisar a influência da capacidade de rede e do compartilhamento de informações e de conhecimento na inovação aberta em startups relacionadas com grandes empresas. Este estudo está alinhado com o fluxo da pesquisa sobre recursos compartilhados em RIOs, atendendo chamadas da literatura. Usman e Vanhaverbeke (2017) destacam a dependência das startups de parceiros externos, mas pouco se sabe sobre quais mecanismos utilizam para gerenciar suas parcerias externas.

Uma questão voltada à sobrevivência das startups é - impacto das suas escolhas estratégicas, o que ainda é pouco investigado. Spender et al. (2017) destacam a necessidade de uma análise mais aprofundada da inovação aberta e das práticas de gestão das startups em RIOs. Em âmbito nacional, ainda se carece de estudos sobre a relação de práticas de inovação aberta entre grandes empresas e startups, o que figura uma oportunidade de pesquisa.

Apesar de pesquisas terem investigado antecedentes da inovação aberta de empresas, há incentivos em ampliar os estudos (Lefebvre et al., 2013; Radziwon, \& Bogers, 2019), em especial sobre inovação aberta em pequenas empresas, como de startups (Spender et al., 2017; Radziwon, \& Bogers, 2019). Embora estudos exaltem a importância da capacidade de rede, a maioria não investigou escalas multidimensionais, tampouco abordagens de mensuração dessa capacidade vinculada a modelos empíricos (Hagedoorn et al., 2006; Parida et al., 2017).

Dessa forma, essa pesquisa estende a literatura relacionada à capacidade de rede e fatores de sucesso organizacional (Walter et al., 2006; Parida et al., 2017; Mitrega et al., 2017), ao averiguar sua influência sobre a inovação aberta. O estudo também contribui para a literatura contábil ao investigar antecedentes e consequentes do compartilhamento de informações e conhecimento em RIOs, destacando os benefícios advindos das interações sociais para o fortalecimento de relações interorganizacionais.

Contribuições práticas e sociais advêm de novos entendimentos sobre o compartilhamento de informações e conhecimento, seus direcionadores e consequentes em RIOs. O gerenciamento de relacionamentos permite que as empresas alinhem sua base de recursos com suas necessidades de inovação aberta, uma vez que essa é caracterizada como mais desafiadora se comparada à inovação fechada (Wu et al., 2019).
Altas taxas de descontinuidade de RIOs têm sido imputadas a problemas de gerenciamento (Parida et al., 2017), logo investigar implicações da capacidade de rede em startups é importante à competitividade e sobrevivência em redes, uma vez que as práticas de inovação aberta das startups são consideradas seu ativo estratégico na obtenção de vantagem competitiva (Singh et al., 2019). Ao identificar influências positivas das dimensões da capacidade de rede como antecedentes destas relações, aponta-se onde startups devem centrar seus esforços para gerenciar o desequilíbrio de poder em RIOs com grandes empresas.

\section{Revisão Teórica e Hipóteses}

\subsection{Capacidade de rede e inovação aberta}

A capacidade de rede pode ser definida como a capacidade orientada para a gestão de relacionamentos em todas as suas etapas de desenvolvimento (Mitrega et al., 2017). Segundo Walter et al. (2006), a capacidade de rede inclui quatro dimensões: conhecimento do parceiro; habilidades de relacionamento; coordenação entre empresas colaboradoras; e comunicação interna. O conhecimento do parceiro permite 0 gerenciamento de situações específicas, as quais podem lidar com instabilidades em suas relações e estabilizar a posição da empresa sempre que necessário (Walter et al., 2006; Partanen et al., 2020).

As habilidades relacionais são voltadas ao gerenciamento de conflitos, senso de justiça, autorreflexão e empatia em relação ao parceiro (Walter et al., 2006). A coordenação entre empresas abrange fronteiras e limites que conectam a empresa a outras empresas (Walter et al., 2006). A comunicação interna traduz-se na comunicação entre os indivíduos da própria empresa e tem a capacidade de assimilar as informações distribuídas nas redes, reduzir a assimetria informacional interna e obter o aprendizado interno com as parcerias (Walter et al., 2006).

Empresas com capacidade de se manterem em relacionamentos, com conhecimento do parceiro, coordenação e comunicação interna competentes, podem elevar sua aptidão de enfrentar riscos e alavancar a inovação (Parida \& Örtqvist, 2015). Com a capacidade de rede, a empresa identifica parceiros usando as habilidades relacionais, coordena relacionamentos e acede o conhecimento sobre o parceiro, gerenciando e estimulando uma atitude inovadora conjunta (Parida et al., 2017). A capacidade de rede proporciona benefícios mútuos aos parceiros, ampara o risco de aprender e ser aprendida nas relações (Walter et al., 2006).

Nessa direção, Chesbrough (2003) propôs um novo modelo de inovação, a inovação aberta (open innovation), definindo-a como o uso de ideias e recursos internos e 
externos para alavancar a inovação, ou seja, tornar mais flexíveis os limites da organização, derrubando barreiras para o desenvolvimento de novos produtos e processos via interação com parceiros. Chesbrough (2003) pondera que os recursos e conhecimentos úteis à inovação estão distribuídos, não sendo possível que a organização os reproduza sozinha, o que instiga identificar e explorar fontes externas. No entanto, um dos desafios das empresas que aderem à inovação aberta é como reduzir - risco do engajamento externo (Kaufmann \& Shams, 2015). Tal inovação depende do gerenciamento eficiente de relacionamentos com parceiros externos (Popa et al., 2017).

A formação e o gerenciamento de relacionamentos externos direcionam as empresas a buscarem novas áreas de cooperação, oportunizando a inovação aberta (Ritter \& Gemünden, 2003). Usman e Vanhaverbeke (2017) destacam que a gestão de redes de parcerias é crucial para o sucesso de startups em ambientes de inovação aberta. Porém, pequenas empresas, como startups, estão mais propensas a riscos relacionais de inovação aberta, sendo uma preocupação que os gestores dessas devem ter (Dushnitsky \& Shaver, 2009). Por conseguinte, startups se mostram menos dispostas a compartilhar dados do seu negócio quando não dispõem de um sistema eficaz de proteção da sua inovação (Dushnitsky \& Shaver, 2009).

Estudos prévios entre capacidade de rede e inovação atestam a relação (Mitrega et al., 2017; Parida et al., 2017; Rakthai et al., 2019; Costa \& Didonet, 2020). Parida et al. (2017) e Mitrega et al. (2017) observaram relação positiva da capacidade de rede com a inovação de produtos. Rakthai et al. (2019) identificaram relação positiva da capacidade de rede com a capacidade de inovação em empresas que passaram por processo de incubação. Costa e Didonet (2020) observaram que a capacidade de rede foi o fator que se sobressaiu dentre os impactos positivos na inovação em marketing.

A capacidade de rede também se mostrou positivamente relacionada com a criação de conhecimento e inovação (Ritter \& Gemünden, 2003; Parida et al., 2017). Yuan (2019) encontrou evidências no contexto chinês de que a capacidade de rede da empresa focal tem efeito direto na inovação colaborativa do cluster. Asemokha et al. (2020) encontraram relação positiva entre a capacidade de rede e a inovação do modelo de negócios, além de mediação da inovação do modelo de negócios na relação entre capacidade de rede e desempenho internacional.

Desta maneira, tendo a inovação aberta como uma variável voltada também ao relacionamento externo, presume-se que empresas com capacidade de rede detêm capacidade de gerenciamento desta variável. Assim, assume-se que:

$\mathrm{H}_{1}$ : A capacidade de rede tem influência positiva na inovação aberta de startups.

\subsection{Compartilhamento de informações e conhecimento e inovação aberta}

O compartilhamento de informações e conhecimento são formas de atividades comunicativas e colaborativas, tanto no âmbito intraorganizacional como interorganizacional (Trkman \& Desouza, 2012). O compartilhamento de informações pode criar conhecimento e aprendizagem nas relações (Cheng, 2011). O compartilhamento de conhecimento implica trocas de experiências, conhecimentos e habilidades (Lin, 2007). Ambos são considerados importantes propulsores de inovação (Belso \& Diez, 2018; Kremer et al., 2019).

No âmbito intraorganizacional, o compartilhamento ocorre entre os indivíduos da própria organização e é importante para o desenvolvimento de novas ideias e inovações (Kremer et al., 2019). No contexto interorganizacional, o compartilhamento de informações e de conhecimento contribui para aumentar a capacidade de inovação das empresas (Belso \& Diez, 2018).

Uma troca recíproca de informações aplica-se na cooperação voltada à inovação aberta, com opções positivas para ambas as partes (Chesbrough, 2003). Ao compartilharem informações, as organizações passam a desenvolver ideias, conhecimentos e inovações conjuntas, resultando na inovação aberta. Chesbrough (2003) destaca que um impulsionador da inovação aberta é o fluxo bidirecional de informações. Rakthai et al. (2019) apontam que a inovação aberta necessita da troca mútua de informações entre departamentos internos e organizações externas. Beuren et al. (2020) encontraram relação positiva entre compartilhamento de informações e inovação colaborativa em cooperativas com alianças estratégicas.

Outra variável que impacta na inovação aberta é o compartilhamento de conhecimento, que, por ser uma informação processada e considerado valioso (Trkman \& Desouza, 2012), muitas vezes as empresas possuem receio de compartilha-lo. Embora apresente riscos de má fé e uso indevido do conhecimento compartilhado (Trkman \& Desouza, 2012), compartilhar conhecimento pode ser importante na inovação aberta (Gupta \& Polonsky, 2014), uma vez que é o recurso central da inovação aberta (Gupta \& Polonsky, 2014).

Consistente com a TDR, que prevê que os RIOs são estabelecidos à medida que organizações necessitam também de recursos externos, dada a dependência do ambiente externo para se manterem vivas no mercado (Pfeffer \& Salancik, 1978), é relevante que haja compartilhamento de conhecimento, pois somente assim será possível alcançar os objetivos comuns, como 
a inovação colaborativa e a eficiência das operações (Trkman \& Desouza, 2012).

Pesquisas apontam que $\circ$ compartilhamento de conhecimento entre empresas proporciona aprendizado mútuo e permite que trabalhem juntas para criar valor e inovações (Tan et al., 2016; Singh et al., 2019). Lin (2007) destaca o compartilhamento de conhecimento como essencial à aprendizagem, por permitir criação de atividades de inovação de mercado. Singh et al. (2019), ao investigarem a relação entre práticas de compartilhamento de conhecimento e inovação aberta, encontraram que as empresas que compartilham conhecimento mais intensamente possuem inovação aberta mais eficiente. Hameed et al. (2021) observaram influência positiva do conhecimento externo no desempenho de inovação aberta em hotéis do Paquistão.

No âmbito de relacionamentos de startups, supõese que haja necessidade de compartilharem suas ideias e conhecimento para conseguirem firmar esses relacionamentos. $O$ compartilhamento acontece geralmente quando há interesses mútuos, ou seja, quando há necessidade de receber algo em troca (lpe, 2003). Apesar de terem características distintas, grandes empresas e startups podem ser complementares, visto que uma tem o que é limitado em outra (Kohler, 2016). Pelo exposto, presume-se que o compartilhamento de informações e conhecimento favorece a cooperação entre parceiros, estimulando novas ideias/tecnologias e projetos colaborativos. Assim, pressupõe-se que:

$\mathrm{H}_{2}$ : O compartilhamento de informações tem influência positiva na inovação aberta de startups.

$\mathrm{H}_{3}$ : O compartilhamento de conhecimento tem influência positiva na inovação aberta de startups.

\subsection{Efeitos do compartilhamento de informações $e$ conhecimento entre capacidade de rede e inovação aberta}

A TDR aponta que todas as organizações dependem de algum recurso externo (Pfeffer \& Salancik, 1978), sendo essa dependência responsável pela criação de RIOs. A literatura sugere que as empresas mais dependentes de recursos externos são as pequenas empresas, por terem limitação de recursos (Parida et al., 2017) e necessidade de estabelecer relações com parceiros para progredirem no mercado (Parida \& Örtqvist, 2015; Parida et al., 2017). Nesse sentido, Morrissey e Pittaway (2006) advertem que essas necessitam de habilidades relacionais e confiança no gerenciamento de seus relacionamentos, para evitar assimetria de poder e dependência demasiada.

Tehseen e Sajilan (2016) analisaram a competência de rede sob a visão baseada em recursos e a TDR, e concluíram que a competência de rede é uma importante competência organizacional, que favorece principalmente pequenas empresas. Hagedoorn et al. (2006) sugerem que empresas podem avançar sua posição na rede quando detêm capacidade de rede. As empresas tendem a se sentirem mais confiantes frente à cooperação com parceiros quando percebem um nível adequado de controle e gestão do relacionamento (Das \& Teng, 1998). Esses autores apontam que para assegurar o cumprimento dos objetivos e criar confiança na colaboração entre parceiros é necessário um gerenciamento eficaz.

Com a capacidade de rede, a empresa gerencia e estimula uma atitude inovadora conjunta (Parida et al., 2017), sendo uma variável importante frente ao desafio de diminuir o risco de engajamento externo que as pequenas empresas enfrentam ao aderir à inovação aberta (Kaufmann \& Shams, 2015).

Estudos sobre capacidade de rede e desempenho (inter) organizacional devem considerar possíveis variáveis intervenientes/ mediadoras, visto que existem diversos fatores que contribuem para tal relação (Fang et al., 2019; Yuan, 2019). Fang et al. (2019) sugerem que - impacto da capacidade de rede no desempenho inovador é exercido pelo efeito mediador dos recursos nas configurações de rede. Yuan (2019) encontrou que a capacidade de rede das empresas focais afeta a inovação colaborativa do cluster por meio da integração de rede dupla; e que a dinâmica do ambiente e a capacidade de integração do conhecimento das empresas do cluster têm efeito mediador na relação entre a capacidade de rede das empresas centrais e a inovação colaborativa do cluster.

A importância do compartilhamento de informações para um relacionamento externo bem-sucedido foi destacado por Trkman e Desouza (2012). Ritter e Gemünden (2003) salientam que é possível que a empresa obtenha informações por meio da formação de relacionamentos externos, o que leva a novos horizontes de cooperação e possibilita a inovação aberta. O compartilhamento de conhecimento também é importante à inovação aberta (Gupta \& Polonsky, 2014).

No entanto, atividades de inovação aberta podem não apresentar vantagens se forem apenas baseadas em relações transacionais e distantes dos parceiros, principalmente no contexto de pequenas empresas (Partanen et al., 2020). Assim, empresas melhor conectadas com seus parceiros podem obter benefícios de redes de inovação por meio do compartilhamento de conhecimento entre si (Fang et al., 2019).

As práticas de compartilhamento de conhecimento já se mostraram mediadoras da relação entre o valor do conhecimento da alta administração e a inovação aberta (Singh et al., 2019). Assim, no presente estudo presume- 
se haver efeito mediador do compartilhamento de informações e conhecimento na relação entre capacidade de rede e inovação aberta. Tal proposição decorre das evidências de que a capacidade de rede influencia - compartilhamento de informações e conhecimento (Ritter \& Gemünden, 2003; Partanen et al., 2020) e que esses compartilhamentos influenciam a inovação aberta (Chesbrough, 2003; Gupta \& Polonsky, 2014; Singh et al., 2019). Desse modo, pressupõe-se que:

H4: O compartilhamento de informações tem efeito mediador na relação entre capacidade de rede e inovação aberta de startups.

H5: O compartilhamento de conhecimento tem efeito mediador na relação entre capacidade de rede e inovação aberta de startups.

Respaldado na literatura, variáveis de controle foram adicionadas ao modelo. Autores destacam que programas de aceleração, competição e incubação propiciam acesso ao mercado às startups. Aceleradoras combinam startups empreendedoras com grandes empresas, que buscam apoiar o desenvolvimento e a aceleração de startups por meio de capacitação, serviços de apoio, inclusive abre oportunidades de acesso a mercados (Kohler, 2016). Já programas de competição oferecem mentorias, inserem startups em competições, além da oportunidade de estabelecer contatos com empresas, empreendedores e investidores, de modo que grandes empresas conseguem identificar inovações externas potencialmente benéficas para seus negócios (Urbaniec \& Zur, 2020). Por fim, incubadoras possuem como atributo central o fornecimento de oportunidades de rede para as startups incubadas formarem relacionamentos de cooperação com outras empresas (Sá \& Lee, 2012).

A literatura destaca ainda que a quantidade de parceiros pode impactar na inovação aberta, visto que uma maior quantidade de parceiros pode fornecer ideias e recursos benéficos à inovação, mas uma quantidade excessiva de parceiros externos pode prejudicar o desempenho da inovação (Laursen \& Salter, 2006).

$\mathrm{Na}$ Figura 1 apresentam-se $\circ$ modelo conceitual da pesquisa e as hipóteses.

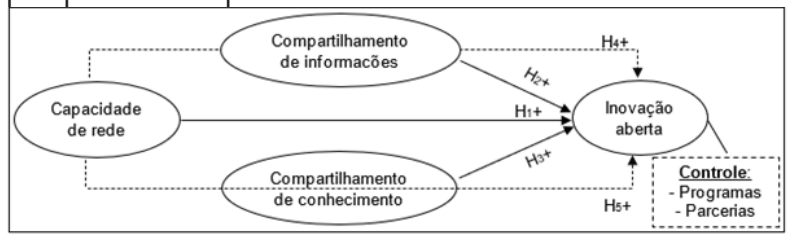

Nota: As setas pontilhadas referem-se às hipóteses de mediação. Figura 1. Modelo teórico da pesquisa

Pela Figura 1, o modelo teórico da pesquisa prevê relação positiva entre capacidade de rede e inovação aberta, entre compartilhamento de informações e inovação aberta, e entre compartilhamento de conhecimento e inovação aberta. Além de testar as relações diretas entre as variáveis mencionadas, propõe identificar o efeito mediador do compartilhamento de informações e de conhecimento. Variáveis de controle também integram o modelo.

\section{Procedimentos Metodológicos}

\subsection{População e amostra}

Uma survey foi realizada com startups ranqueadas na plataforma 100 Open Startups, no período de 2016 a 2020. Esse ranking iniciou-se em 2016, o que justifica o corte temporal inicial, enquanto o término limita-se ao ano da operacionalização desta pesquisa. A opção dessas startups decorre do fato de serem empresas engajadas em inovação aberta e manterem relações com grandes empresas. $O$ mapeamento das startups foi realizado no site openstartups, que disponibiliza a startup ranqueada, categoria e pontos alcançados em open innovation. Portanto, a população da pesquisa compreende as 324 startups ranqueadas no período de 2016 a 2020 na plataforma 100 Open Startups.

A partir desta listagem, buscou-se estabelecer contato com profissionais em cargos mais estratégicos (ex: CEO, Gestores) na rede profissional Linkedln sob a premissa de que estejam engajados na gestão dos RIOs de sua empresa. Enviou-se convite para conexão aos 837 profissionais identificados e, aos 471 que aceitaram 0 convite, foi enviado o link do questionário na plataforma QuestionPro. Cuidados foram tomados para não haver polarização de respondentes de uma única startup, delimitando-se no máximo três respondentes por empresa. No período de dezembro de 2020 a janeiro de 2021 obteve-se um total de 144 respostas válidas (17,2\%).

\subsection{Construtos e instrumento de pesquisa}

Os construtos e variáveis deste estudo foram aferidos por meio de um questionário (Apêndice $A$ ), alicerçados em estudos anteriores e validados em pesquisas prévias. $O$ questionário compreende assertivas de escalas múltiplas, mensuradas em escala tipo Likert de sete pontos.

A capacidade de rede foi aferida pelo instrumento de pesquisa de Partanen et al. (2020), adaptado de Walter et al. (2006). Buscou-se aferir o grau de disponibilidade de quatro dimensões da capacidade de rede: (i) coordenação entre empresas; (ii) habilidades de relacionamento; (iii) conhecimento do parceiro; e (iv) comunicação interna. Os respondentes foram indagados até que ponto as assertivas se aplicam na organização em relação à forma, cuidado e uso dos relacionamentos com os seus principais parceiros de inovação aberta (grandes empresas que selecionaram a sua startup para 
desenvolvimento de inovações), na escala de 1 = não se aplica a 7 = se aplica completamente.

No construto compartilhamento de informações utilizaramse assertivas do instrumento de pesquisa de Cheng (2011) e a assertiva "Nós e nossos parceiros comerciais e/ou outras partes externas trocamos informações que ajudam no planejamento dos negócios" de Tan et al. (2016). Questionou-se quanto ao grau de concordância com cada uma das assertivas sobre compartilhamento de informações no âmbito do relacionamento da sua organização com os seus principais parceiros de inovação aberta (grandes empresas que selecionaram a sua startup para desenvolvimento de inovações), na escala de 1 = discordo totalmente a 7 = concordo totalmente.

Para o compartilhamento de conhecimento adaptou-se o instrumento de pesquisa de Wang e Hu (2020), acerca da relação das startups com seus parceiros (grandes empresas). As assertivas originais buscavam levantar a realidade de relacionamentos com membros da cadeia de suprimentos. Indagou-se os respondentes sobre 0 grau de concordância com cada uma das assertivas sobre compartilhamento de conhecimento no âmbito do relacionamento da organização com seus principais parceiros de inovação aberta (grandes empresas que selecionaram a sua startup para desenvolvimento de inovações), na escala de $1=$ discordo totalmente a $7=$ concordo totalmente.

No construto inovação aberta, utilizou-se o instrumento de pesquisa de Hameed et al. (2018). Indagou-se sobre - grau de concordância com cada uma das assertivas relativas ao desempenho da inovação aberta (com grandes empresas que selecionaram a sua startup para desenvolvimento de inovações) na organização, na escala de 1 = discordo totalmente a 7 = concordo totalmente.

$\mathrm{Na}$ premissa de que características organizacionais (quantidade de relacionamentos e participação em programas de aceleração, competição ou incubação) podem afetar a inovação aberta (Laursen \& Salter, 2006; Sá \& Lee, 2012; Kohler, 2016; Urbaniec \& Zur, 2020), variáveis de controle foram inseridas no questionário. A quantidade de relacionamentos, operacionalizada como variável contínua, foi aferida questionando-se: "Quantas parcerias aproximadamente sua organização possui?". Quanto aos programas, questionou-se: "Você já participou de algum programa de aceleração, incubação ou competição?". O resultado para esta questão foi: sim $73,6 \%$ e não $26,4 \%$.

\subsection{Procedimentos de análise dos dados}

Procedimentos de análise descritiva e modelagem de equações estruturais (SEM-SmartPLS), estimadas por Mínimos Quadrados Parciais (Partial Least Squares - PLS), foram utilizados. Na SEM-PLS, as relações diretas foram analisadas pelos coeficientes de caminho (path), e as relações indiretas pelos coeficientes indiretos totais (Hair et al., 2017). Na análise da mediação, foram seguidos os preceitos de Hair et al. (2017) e Bido e Silva (2019), de que a variável antecedente deve influenciar a mediadora e a mediadora deve influenciar a consequente. Bido e Silva (2019) frisam que para a confirmação de mediação total, o efeito direto não deve ter significância, e o indireto deve ser significante.

A capacidade de rede foi tratada como um construto de segunda ordem. Desse modo, seus construtos de primeira ordem (coordenação entre empresas, habilidades de relacionamento, conhecimento do parceiro) foram tratados com abordagem de repetição dos indicadores, do tipo reflexivo-reflexivo.

\subsection{Testes de vieses}

O método de coleta de dados pode levar ao viés do método comum (Common Method Bias), característico dos estudos de recorte transversal, no qual as respostas são coletadas em um mesmo período e por uma mesma fonte (Podsakoff et al., 2003). Para mitigar tal problema, alertou-se os respondentes de que não há respostas certas ou erradas, de forma que respondessem conforme sua percepção. Também se aplicou o teste de fator único de Harman, que verifica se os dados possuem representativas limitações de viés do método comum, seguindo os pressupostos de Podsakoff et al. (2003). Os resultados do teste indicaram que a variância total explicada foi de $64,86 \%$, sendo que o primeiro fator explicou apenas $32,34 \%$ da variância total, portanto, nenhum fator isolado representa individualmente grande parte da variância $(>50 \%)$, e que os dados da pesquisa não apresentam limitações relativas ao viés de método comum.

Também foram averiguadas possíveis distorções na amostra por meio do teste do viés de não resposta, utilizando o critério de comparação firts-lasts, devido à impossibilidade de identificar aqueles que optaram por não responder o questionário (Mahama \& Cheng, 2013). Desse modo, aplicou-se o teste † para amostras independentes como forma de comparar as respostas das assertivas do estudo dos 10\% primeiros e últimos respondentes (14 primeiros e últimos). Ao nível de significância de $5 \%$, não foram encontradas diferenças significativas entre os grupos. Assim, presume-se que $\circ$ viés de não respostas não é representativo (Mahama \& Cheng, 2013). Em conjunto, os resultados dos testes mitigam a apreensão de possíveis vieses que pudessem reproduzir ruídos na análise dos dados.

O método de coleta de dados pode levar ao viés do método comum (Common Method Bias), característico dos estudos de recorte transversal, no qual as respostas 
são coletadas em um mesmo período e por uma mesma fonte (Podsakoff et al., 2003). Para mitigar tal problema, alertou-se os respondentes de que não há respostas certas ou erradas, de forma que respondessem conforme sua percepção. Também se aplicou o teste de fator único de Harman, que verifica se os dados possuem representativas limitações de viés do método comum, seguindo os pressupostos de Podsakoff et al. (2003). Os resultados do teste indicaram que a variância total explicada foi de $64,86 \%$, sendo que o primeiro fator explicou apenas $32,34 \%$ da variância total, portanto, nenhum fator isolado representa individualmente grande parte da variância (>50\%), e que os dados da pesquisa não apresentam limitações relativas ao viés de método comum.

Também foram averiguadas possíveis distorções na amostra por meio do teste do viés de não resposta, utilizando o critério de comparação firts-lasts, devido à impossibilidade de identificar aqueles que optaram por não responder 0 questionário (Mahama \& Cheng, 2013). Desse modo, aplicou-se $\circ$ teste $\dagger$ para amostras independentes como forma de comparar as respostas das assertivas do estudo dos $10 \%$ primeiros e últimos respondentes (14 primeiros e últimos). Ao nível de significância de 5\%, não foram encontradas diferenças significativas entre os grupos. Assim, presume-se que o viés de não respostas não é representativo (Mahama \& Cheng, 2013). Em conjunto, os resultados dos testes mitigam a apreensão de possíveis vieses que pudessem reproduzir ruídos na análise dos dados.

\section{Descrição e Análise dos Resultados}

\subsection{Perfil das empresas e dos respondentes}

Os respondentes, em sua maioria $(80,56 \%)$ são do gênero masculino e $68,06 \%$ possuem idade até 40 anos. Grande parte $(40,97 \%)$ possui pós-graduação em nível de especialização e/ou Master of Business Administration (MBA), sendo que $29,86 \%$ têm formação em administração/negócios/gestão. Quanto ao cargo na empresa, a maioria está na alta gerência $(59,03 \%)$ e a menos de 1 ano (30,56\%). Este perfil é condizente com a realidade das startups, por serem empresas novas e iniciarem com poucos fundadores de alto nível e multidisciplinares (Ries, 2011).

Sobre as startups, metade está em funcionamento de 5 a 7 anos (50\%), seguido de 1 a 4 anos (25\%); e $43,06 \%$ dispõem de menos de 20 funcionários. Esses números condizem com a realidade de startups, que geralmente são jovens, atuam com alto grau de tecnologia e equipes enxutas (Ries, 2011). Quanto ao estágio em que se encontram as startups, foi apontado que $84,72 \%$ iá está nas fases finais (clientes pagantes, lançados no mercado) da inovação, quando podem aproveitar os canais de comercialização que as grandes empresas abrem (Hogenhuis et al., 2016). De modo geral, as características das startups respondentes são convergentes com outros estudos realizados no mesmo contexto (Ries, 2011; Hogenhuis et al., 2016).

\subsection{Modelo de mensuração}

No modelo de mensuração são verificados os índices de confiabilidade (interna e composta) e validade (discriminante e convergente) dos construtos (Hair et al., 2017). Para a confiabilidade dos indicadores dos construtos são recomendados valores superiores a 0,70, mas cargas entre 0,40 e 0,70 só devem ser excluídas se levarem ao aumento da Variância Média Extraída (AVE) e da Confiabilidade Composta (CR) (Hair et al., 2017). Assim, excluiu-se uma assertiva de capacidade de rede (na comunicação interna - Cl2) para ajuste do modelo. Na Tabela 1, apresentam-se os resultados do modelo.

Tabela 1. Confiabilidade e validade do modelo de mensuração

\begin{tabular}{|c|c|c|c|c|c|c|}
\hline Variáveis latentes & \multirow{2}{*}{1} & \multirow{2}{*}{2} & \multirow{2}{*}{3} & \multirow{2}{*}{4} & \multirow{2}{*}{5} & \multirow{2}{*}{6} \\
\hline Indicadores & & & & & & \\
\hline 1. Capacidade de rede & 0,640 & & & & & \\
\hline $\begin{array}{l}\text { 2. Compartilhamento de } \\
\text { Informações }\end{array}$ & 0,589 & 0,776 & & & & \\
\hline $\begin{array}{l}\text { 3. Compartilhamento de } \\
\text { Conhecimento }\end{array}$ & 0,487 & 0,424 & 0,841 & & & \\
\hline 4. Inovação Aberta & 0,427 & 0,398 & 0,551 & 0,756 & & \\
\hline 5. Programas & 0,126 & 0,244 & 0,088 & 0,016 & 1 & \\
\hline 6. Parcerias & 0,123 & 0,115 & 0,107 & 0,000 & $-0,034$ & 1 \\
\hline Mediana & 6 & 6 & 5 & 5 & & \\
\hline Média & 5,87 & 5,35 & 5,00 & 5,17 & & \\
\hline Desvio Padrão & 1,19 & 1,54 & 1,74 & 1,61 & & \\
\hline AVE $>0,50$ & 0,573 & 0,602 & 0,707 & 0,571 & 1 & 1 \\
\hline Alfa de Cronbach $>0,70$ & 0,852 & 0,781 & 0,781 & 0,874 & 1 & 1 \\
\hline$C R>0,70$ & 0,842 & 0,857 & 0,879 & 0,903 & 1 & 1 \\
\hline
\end{tabular}

Nota: VIF (Variance Inflation Factors): Capacidade de rede $=1,717$; Compartilhamento de informações = 1,671; Compartilhamento de conhecimento = 1,366; Programas = 1,068; Parcerias = 1,024.

Fonte: dados da pesquisa.

Os resultados da análise descritiva denotam que os respondentes percebem alta presença das variáveis desta pesquisa (ficando acima do ponto médio 4), principalmente da capacidade de rede (média 5,87; mediana 6).

Todas as variáveis observadas apresentaram valores de alfa de Cronbach e CR maiores que 0,70, o que indica que as assertivas em seu conjunto são confiáveis. Quanto à validade convergente, a AVE demonstra que cada variável explica mais da metade da variância de seus indicadores, portanto, superior ao mínimo estabelecido pela literatura para atestar a validade convergente das variáveis. A validade discriminante foi analisada pelo 
critério de Fornell-Larcker, em que todas as variáveis apresentam valores superiores aos coeficientes da matriz de correlação, o que sugere validade discriminante aceitável.

Como forma de assegurar a ausência de multicolinearidade entre as variáveis latentes realizou-se a análise dos indicadores de Variance Inflation Factors (VIF), cujos valores devem ser inferiores a 3 (Hair et al., 2017). Confirma-se ausência de multicolinearidade entre as variáveis, que apresentaram valores abaixo de 2. Portanto, as análises indicam que o modelo de mensuração é adequado, o que permite realizar a análise das relações estruturais.

\subsection{Modelo estrutural e teste das hipóteses}

Para testar o modelo estrutural, utilizou-se a técnica bootstraping, tendo como parâmetros 5.000 reamostragens (samples) e 5.000 interações, intervalo de confiança com enviesamento corrigido e acerelado (bias-corrected and accelerated) e teste bicaudal ao nível de significância de 10\% (Hair et al., 2017). Na Tabela 2 apresenta-se a análise de caminhos, coeficiente beta ( $\beta$ ), t-value, $p$-value e decisão para cada hipótese. Considerou-se o coeficiente de determinação de Pearson $\left(\mathrm{R}^{2}\right)$ e Relevância Preditiva, pelo indicador de Stone-Geisser $\left(Q^{2}\right)$, para atestar a validação e precisão do modelo.

Tabela 2. Resultados do modelo estrutural: teste das hipóteses

\begin{tabular}{|c|c|c|c|c|c|}
\hline & $\begin{array}{l}\text { Capacidade de rede -> } \\
\text { Compartilhamento de } \\
\text { Informaçōes }\end{array}$ & 0,589 & 9,101 & $0,000 *$ & \\
\hline & $\begin{array}{l}\text { Capacidade de rede.-> } \\
\text { Compartilhamento de } \\
\text { Conhecimento }\end{array}$ & 0,487 & 6,022 & $0,000^{*}$ & \\
\hline $\mathrm{H}_{1}$ & $\begin{array}{l}\text { Capacidade de rede -> } \\
\text { Inovação Aberta }\end{array}$ & 0,144 & 1,419 & 0,156 & Rejeitar \\
\hline $\mathrm{H}_{2}$ & $\begin{array}{l}\text { Compartilhamento de } \\
\text { Informações - I Inovação Aberta }\end{array}$ & 0,161 & 2,064 & $0,039 * *$ & $\begin{array}{c}\text { Não } \\
\text { Rejeitar }\end{array}$ \\
\hline $\mathrm{H}_{3}$ & $\begin{array}{c}\text { Compartilhamento de } \\
\text { Conhecimento }>>\text { Inovação } \\
\text { Aberta }\end{array}$ & 0,429 & 6,432 & $0,000 *$ & $\begin{array}{l}\text { Não } \\
\text { Rejeitar }\end{array}$ \\
\hline $\mathrm{H}_{4}$ & $\begin{array}{c}\text { Capacidade de rede -> } \\
\text { Compartilhamento de } \\
\text { Informaçōes }>>\text { Inovação Aberta }\end{array}$ & 0,095 & 1,935 & $0,053 * * *$ & $\begin{array}{l}\text { Não } \\
\text { Rejeitar }\end{array}$ \\
\hline $\mathrm{H}_{5}$ & $\begin{array}{c}\text { Capacidade de rede -> } \\
\text { Compartilhamento de } \\
\text { Conhecimento }->\text { Inovação } \\
\text { Aberta }\end{array}$ & 0,209 & 4,112 & $0,000 *$ & $\begin{array}{c}\text { Não } \\
\text { Rejeitar }\end{array}$ \\
\hline $\mathrm{Cl}$ & Programas -> Inovação Aberta & $-0,083$ & 1,370 & 0,171 & Rejeitar \\
\hline C2 & Parcerias -> Inovação Aberta & $-0,086$ & 1,426 & 0,154 & Rejeitar \\
\hline
\end{tabular}

Nota 1: Significante ao nivel de ${ }^{*} p<0,01 ;{ }^{* *} p<0,05 ;{ }^{* * *} p<0,10$.

Nota2: $\mathrm{Cl}=$ controle $1 ; \mathrm{C2}=$ controle 2 .

Fonte: dados da pesquisa.

A $H_{1}$ postulou que a capacidade de rede tem influência direta e positiva sobre a inovação aberta. Apesar da relação positiva, os resultados não apresentaram significância estatística $(p>0,10)$. Desse modo, não se pode afirmar que a capacidade de rede influencia diretamente na inovação aberta. Infere-se que variáveis intervenientes podem estar presentes e ter impactos nessa relação. Por outro lado, influências positivas foram encontradas entre capacidade de rede e compartilhamento de informações $(\beta=0,589$; $p<0,01)$ e entre capacidade de rede e compartilhamento de conhecimento $(\beta=0,487 ; p<0,01)$.

A $\mathrm{H}_{2}$, que previa relação direta e positiva entre $\circ$ compartilhamento de informações e a inovação aberta, foi suportada $(\beta=0,161 ; p<0,05)$. Isso indica que $\circ$ compartilhamento de informações interorganizacional impacta de maneira direta e positiva na inovação aberta. Da mesma forma, a relação direta do compartilhamento de conhecimento com inovação aberta permite a aceitação da $\mathrm{H}_{3}(\beta=0,429 ; p<0,10)$, o que sugere que - compartilhamento de conhecimento reflete direta e positivamente na inovação aberta.

As hipóteses $\mathrm{H}_{4}$ e $\mathrm{H}_{5}$ previam mediação do compartilhamento de informações e do compartilhamento conhecimento na relação entre capacidade de rede e inovação aberta. Não se observou relação direta entre a capacidade de rede e a inovação aberta, mas indireta ao inserir a variável mediadora, assim considera-se mediação total em ambas as hipóteses. Esses resultados fornecem evidências para a não rejeição das hipóteses $\mathrm{H}_{4}$ e $\mathrm{H}_{5}$. Portanto, a capacidade de rede influencia a inovação aberta por meio do compartilhamento de informações $(\beta=0,095 ; p<0,10)$ e conhecimento $(\beta=0,209 ; p<0,01)$.

Propôs-se a inclusão de variáveis de controle ao modelo destacadas pela literatura (Laursen \& Salter, 2006; Sá \& Lee, 2012; Kohler, 2016; Urbaniec \& Zur, 2020) como influentes na inovação aberta. Os resultados não apresentaram significância estatística em relação ao número de parcerias e à inovação aberta $(p>0,10)$, assim como em relação à participação em programas de aceleração, incubação ou competição e a inovação aberta $(p>0,10)$. Desse modo, não houve influência das variáveis de controle no modelo.

Por fim, avaliaram-se os indicadores de ajustes gerais do modelo. Verifica-se que o modelo apresenta um $\mathrm{R}^{2}$ de 0,232 para compartilhamento de informações, 0,343 para compartilhamento de conhecimento e 0,338 para inovação aberta, o que representa poder de explicação de efeito médio e grande, respectivamente. Em relação ao Q², os resultados ficaram acima de zero, compartilhamento de informações 0,195, compartilhamento de conhecimento 0,160 e inovação aberta 0,191 , o que confirma a acurácia do modelo (Hair et al., 2017).

\subsection{Discussão dos resultados}

As discussões são delineadas a partir dos resultados apresentados na Figura 2, que evidencia as relações significantes. 


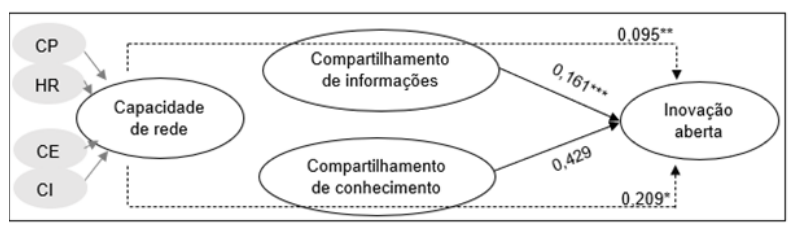

Nota 1: Significante ao nivel de ${ }^{*} p<0,01 ;{ }^{* *} p<0,05 ;{ }^{* *} p<0,10$.

Nota2: Variáveis que compõem a capacidade de rede: $C P=$ conhecimento do parceiro, $H R=$ habilidades de relacionamento, $C E=$ coordenação entre empresas, $\mathrm{Cl}=$ comunicação interna.

Figura 2. Resultados significativos do modelo estrutural

A $\mathrm{H}_{1}$, que previa relação positiva e significativa entre a capacidade de rede e a inovação aberta, foi rejeitada, pois não apresentou significância estatística. Isso difere de Lefebvre et al. (2013), que encontraram relações diretas entre competência de rede e abertura das empresas do setor alimentício da Europa. O estudo do questionário original de capacidade de rede (Walter et al., 2006) identificou relação positiva da capacidade de rede e desempenho de spin-offs. Apesar da capacidade de rede ser vista como uma variável importante para a inovação aberta, por promover acesso a parceiros, impulsionar a abertura e ter capacidade de gerenciamento da rede (Lefebvre et al., 2013; Parida et al., 2017), variáveis intervenientes podem influenciar nessa relação (Fang et al., 2019; Yuan, 2019).

Confirmou-se relação significativa positiva e direta entre capacidade de rede e compartilhamento de informações e conhecimento. Embora não tenha sido formulada como hipótese de pesquisa, pode-se afirmar que a capacidade de rede ampara o compartilhamento de informações e conhecimento nessas relações (lpe, 2003; Trkman \& Desouza, 2012).

De acordo com Walter et al. (2006) e Parida et al. (2017), isso decorre do fato da empresa ser capaz de: (i) coordenar relacionamentos e situações, o que possibilita uma melhor resposta ao compartilhamento entre parceiros; (ii) ter o conhecimento do parceiro, o que aumenta o vínculo e permite o gerenciamento de situações específicas; (iii) possuir habilidades relacionais, que impactam diretamente na capacidade das startups de influenciar trocas pessoais; e (iv) haver comunicação interna, que impacta por ter a capacidade de assimilar as informações distribuídas, obter o aprendizado em relação às parcerias e por mitigar o risco de encaminhar mensagens conflitantes e confusas aos seus parceiros.

A $\mathrm{H}_{2}$, que previa relação positiva e significativa do compartilhamento de informações com a inovação aberta, não foi rejeitada. Os resultados coadunam com os de Chesbrough (2003), Trkman e Desouza (2012), Rakthai et al. (2019), Beuren et al. (2020), de que o compartilhamento de informações possui impactos diretos sobre a inovação aberta. Ao compartilhar informações, tem-se uma cooperação benéfica à inovação aberta (Chesbrough, 2003), uma vez que as organizações passam a desenvolver conhecimentos, ideias e inovações conjuntas. A associação positiva entre compartilhamento de informações e inovação aberta sugere que os sistemas de informação fornecem suporte às parcerias.

Também se encontrou influência direta e positiva do compartilhamento de conhecimento na inovação aberta, o que suporta não rejeitar a $\mathrm{H}_{3}$. Assim, pode-se afirmar que o compartilhamento de conhecimento se destaca no contexto colaborativo investigado, estreitando relações, potencializando relacionamentos e fomentando a inovação. Singh et al. (2019) também encontraram efeitos diretos do compartilhamento de conhecimento na inovação aberta em pequenas e médias empresas dos Emirados Árabes. Esses resultados coadunam com Trkman e Desouza (2012), Gupta e Polonsky (2014), Singh ef al. (2019), de que o compartilhamento de conhecimento é um recurso central da inovação aberta, por propiciar conhecimento às redes de empresas e promover a inovação.

Enquanto formas de atividades colaborativas e comunicativas, os compartilhamentos de informações e de conhecimento são considerados essenciais ao desempenho da inovação aberta. Assim, com a não rejeição das hipóteses $\mathrm{H}_{2}$ e $\mathrm{H}_{3}$ contribui-se para uma linha de pesquisa diferenciada, ao abordar a visão de startups em RIOs e destacar os impactos positivos desses compartilhamentos na inovação aberta. De acordo com Ipe (2003), o compartilhamento acontece geralmente quando há interesses mútuos e, dessa forma, startups necessitam compartilhar conhecimento para conseguir firmar esses relacionamentos de inovação, apesar dos riscos do compartilhamento.

As hipóteses que previam mediação do compartilhamento de informações e do compartilhamento de conhecimento na relação entre a capacidade de rede e a inovação aberta $\left(\mathrm{H}_{4}\right.$ e $\left.\mathrm{H}_{5}\right)$ não foram rejeitadas. Dessa maneira, confirma-se a interação das variáveis intervenientes nessa relação. Ambas as mediações foram propostas com base em estudos que salientam efeitos positivos do compartilhamento de informações e conhecimento em RIOs (Gupta \& Polonsky, 2014; Singh et al., 2019, Beuren et al., 2020).

Se o compartilhamento é necessário para firmar relações entre empresas (lpe, 2003), a capacidade de rede pode ser um antecedente significante na relação, por diminuir o risco do engajamento (Kaufmann \& Shams, 2015). As organizações tendem a se sentir mais confiantes quando percebem um nível de gestão adequado frente às trocas, pois o medo da exploração e a falta de vínculo entre as empresas podem ser empecilhos ao compartilhamento de informações e conhecimento (Das \& Teng, 1998; O'dell \& Grayson, 1998). 
A capacidade de rede, ao propiciar o gerenciamento de situações específicas, vínculo entre empresas e evitar assimetria de poder demasiada entre as partes (Morrissey \& Pittaway, 2006), é benéfica às startups, por impactar no compartilhamento de informações e conhecimento e estimular de maneira indireta a inovação aberta, propiciando maior desempenho.

Tehseen e Sajilan (2016) analisaram a competência de rede sob a lente da TDR e concluíram que esta leva ao sucesso principalmente de pequenas empresas, evidências corroboradas no contexto aqui examinado. Entende-se que o compartilhamento de informações e de conhecimento seja essencial na relação, ao passo que tais compartilhamentos são vistos como recursos centrais à inovação aberta, e a capacidade de rede é essencial por adequar e gerenciar situações e riscos aos quais se expõem as empresas nesses processos abertos.

Como as variáveis de controle do estudo não tiveram impacto no modelo proposto, infere-se que as empresas da amostra se engajam igualmente nos relacionamentos estabelecidos e possuem níveis similares de desempenho de inovação aberta, independentemente da quantidade de relacionamentos e da participação em programas de aceleração, incubação ou competição.

\section{Conclusão}

Sob a lente da TDR, este estudo analisou a influência da capacidade de rede e do compartilhamento de informações e conhecimento na inovação aberta em startups relacionadas com grandes empresas. Os resultados mostraram que a capacidade de rede não possui influência significativa direta sobre a inovação aberta nas startups pesquisadas. Esses achados podem advir das características próprias das startups, bem como do contexto analisado.

O compartilhamento de informações e conhecimento apresentaram influência positiva direta na inovação aberta. Esses resultados também foram encontrados em outros contextos na literatura (Singh et al., 2019; Beuren et al., 2020; Hameed et al., 2021). Os achados da pesquisa indicam que o compartilhamento de informações e conhecimento são antecedentes da inovação aberta, dessa maneira favorecem interação entre as organizações e são oportunos para alcançar os objetivos comuns.

O efeito mediador do compartilhamento de informações e do compartilhamento de conhecimento na relação entre a capacidade de rede e a inovação aberta foi confirmado. Em consonância com a TDR, argumenta-se que, ao deterem capacidade de rede as organizações conseguem reduzir a assimetria de poder entre as partes e o risco do engajamento, e isso impacta de maneira positiva no compartilhamento de informações e de conhecimento. O compartilhamento de informações e de conhecimento, por sua vez, é responsável por firmar esses relacionamentos de inovação aberta e beneficiar a interação entre parceiros.

Conclui-se pelos resultados da pesquisa que a capacidade de rede influencia indiretamente a inovação aberta, por meio do compartilhamento de informações e conhecimento. Os achados indicam que as variáveis investigadas são importantes propulsores do desempenho da inovação aberta, de modo a reduzir riscos e trazer impactos positivos às startups em RIOs.

Este estudo traz implicações à literatura ao revelar que o compartilhamento de informações geradas no âmbito da contabilidade gerencial e compartilhamento de conhecimento auxiliam no apoio à gestão interorganizacional. Ao encontrar variáveis mediadoras significantes na relação entre capacidade de rede e inovação aberta avança-se no entendimento sobre recursos compartilhados em RIOs. Se esses fatores mediadores (compartilhamento de informações e conhecimento) não são salientados, a relação direta entre a capacidade de rede e o desempenho da inovação aberta não é percebida.

Assim, destaca-se a relevância de sistemas de informação que suportam o gerenciamento de RIOs e maior desempenho da inovação aberta. Dessa maneira, expande-se a compreensão do compartilhamento de informações e conhecimento em RIOs (Gupta \& Polonsky, 2014; Singh et al., 2019, Beuren et al., 2020) de startups relacionadas com grandes empresas.

Também se preenche a lacuna observada quanto à inovação aberta no contexto de startups (Cajuela \& Galina, 2020). Enquanto grande parte dos estudos abordou a inovação aberta do ponto de vista de grandes empresas (Popa et al., 2017; Cajuela \& Galina, 2020), este estudo concentrou-se em startups, as quais normalmente dependem de recursos externos para o alcance de seus objetivos. Os achados também trazem implicações sociais, na medida em que fornecem informações sobre RIOs.

Embora ainda recentes, os relacionamentos entre startups e grandes empresas já se mostram necessários (Usman \& Vanhaverbeke, 2017). À vista disso, espera-se que os resultados deste estudo auxiliem os gestores na condução do relacionamento, com o propósito de reduzir assimetrias entre os envolvidos e direcionar para uma relação benéfica de ambas as partes.

Ao apresentar resultados positivos nas relações, apontamse as condições em que startups podem desempenhar com sucesso a inovação aberta, e os impactos que podem trazer as escolhas estratégicas adotadas pelas mesmas, reduzindo a descontinuidade de RIOs. As limitações do estudo podem representar oportunidades para pesquisas futuras. O método survey implica na abordagem 
transversal do problema, assim pesquisas futuras podem realizar: estudos de caso, de forma a esclarecer aspectos que podem intervir na relação da capacidade de rede com a inovação aberta; estudos experimentais, a fim de controlar variáveis e mensurar diferentes cenários.

Os construtos foram adaptados para mensurar os relacionamentos entre grandes empresas e startups na visão das startups, portanto, refletem apenas a percepção destas, o que oportuniza a investigação do outro lado da relação. Estudos futuros podem adaptar o modelo teórico deste estudo para diferentes contextos, a fim de verificar se em outros relacionamentos confirmam-se as relações propostas, como em cadeias de suprimentos, nas relações em incubadoras e parques tecnológicos.

\section{Referências}

Asemokha, A., Torkkeli, L., Faroque, A. R., \& Saarenketo, S. (2020). Business model innovation in international performance: the mediating effect of network capability. International Journal of Export Marketing, 3(4), 290-313.

Belso, J., \& Diez, I. (2018). Firm's strategic choices and network knowledge dynamics: How do they affect innovation? Journal of Knowledge Management, 22(1), 1-20. https://doi.org/10.1 108/JKM-12-2016-0524

Beuren, I. M., Santos, V., Bernd, D. C., \& Pazetto, C. F. (2020). Reflexos do compartilhamento de informações e da inovação colaborativa na responsabilidade social de cooperativas. RBGN: Revista Brasileira de Gestão de Negócios, 22(2), 310-330. https://doi.org/10.7819/ rbgn.v22i 2.4052

Bido, D. S., \& Silva, D. (2019). SmartPLS 3: especificação, estimação, avaliação e relato. Administração: Ensino e Pesquisa, 20(2), 1-31. https://doi.org/10.13058/ raep.2019.v20n2.1545

Cajuela, A. R., \& Galina, S. V. R. (2020). Processos em relacionamentos interorganizacionais para desenvolvimento de capacidade de absorção em startups. Revista de Administração Contemporânea, 24(6), 550-566. https:// doi.org/10.1590/1982-7849rac2020180329

Cheng, J. H. (2011). Inter-organizational relationships and knowledge sharing in green supply chains-Moderating by relational benefits and guanxi. Transportation Research Part E: Logistics and Transportation Review, 47(6), 837 849. https://doi.org/10.1016/i.tre.2010.12.008

Chesbrough, H. W. (2003). Open innovation: The new imperative for creating and profiting from technology. Brighton: Harvard Business Press.

Costa, B. C., \& Didonet, S.R. (2020). Innovation strategy and network capability as marketing innovation enablers. International Journal of Business Innovation and Research, 23(3), 370-383. https://doi.org/10.1504/ IJBIR.2020.110965
Das, T. K., \& Teng, B. S. (1998). Between trust and control: developing confidence in partner cooperation in alliances. Academy of Management Review, 23(3), 491-512. https://doi.org/10.5465/amr. 1998.926623

Dushnitsky, G., \& Shaver, J. M. (2009). Limitations to interorganizational knowledge acquisition: The paradox of corporate venture capital. Strategic Management Journal, 30(10), 1045-1064. https://doi.org/10.1002/smj.781

Fabrício, R. D. S. Jr., Silva, F. R., Simões, E., Galegale, N. V., \& Akabane, G. K. (2015). Strengthening of Open Innovation Model: using startups and technology parks. IFAC-PapersOnline, 48(3), 14-20. https://doi. org/10.1016/j.ifacol.2015.06.051

Fang, G., Zhou, Q., Wu, J., \& Qi, X. (2019). The relationship between network capabilities and innovation performance. Industrial Management \& Data Systems, $119(8)$, 1638-1654. https://doi.org/10.1 108/IMDS-02$2019-0060$

Gupta, S., \& Polonsky, M. (2014). Inter-firm learning and knowledge-sharing in multinational networks: An outsourced organization's perspective. Journal of Business Research, 67(4), 615-622. https://doi.org/10.1016/i. jbusres.2013.02.043

Hagedoorn, J., Roijakkers, N., \& Van Kranenburg, H. (2006). Inter-Firm R\&D Networks: the Importance of Strategic Network Capabilities for High-Tech Partnership Formation. British Journal of Management, 17(1), 39-53. https://doi.org/10.1111/j.1467-8551.2005.00474.x

Hair, J. F.J. Jr., Hult, G. T. M., Ringle, C., \& Sarstedt, M. (2017). A primer on partial least squares structural equation modeling (PLS-SEM) (2nd ed.). Los Angeles: Sage.

Hameed, W. U., Basheer, M. F., lqbal, J., Anwar, A., \& Ahmad, H. K. (2018). Determinants of firm's open innovation performance and the role of R\&D department: an empirical evidence from Malaysian SME's. Journal of Global Entrepreneurship Research, 8(1), 1-20. https:// doi.org/10.1186/s40497-018-0112-8

Hameed, W. U., Nisar, Q. A., \& Wu, H. C. (2021). Relationships between external knowledge, internal innovation, firms' open innovation performance, service innovation and business performance in the Pakistani hotel industry. International Journal of Hospitality Management, 92, 102745. https://doi.org/10.1016/i. ijhm.2020.102745

Hogenhuis, B. N., Van den Hende, E. A., \& Hultink, E.J. (2016). When should large firms collaborate with young ventures? Understanding young firms' strengths can help firms make the right decisions around asymmetric collaborations. Research-Technology Management, 59(1), 39-47. https://doi.org/10.1080/08956308.2016.1117 329 
Ipe, M. (2003). Knowledge sharing in organizations: a conceptual framework. Human Resource Development Review, 2(4), 337-359. https://doi. org/10.1177/1534484303257985

Kaufmann, H. R., \& Shams, S. M. R. (2015). Entrepreneurial challenges in the 21st century: Creating stakeholder value co-creation. Hampshire, UK: Springer/ Palgrave Macmillan.

Kim, M., \& Chai, S. (2017). The impact of supplier innovativeness, information sharing and strategic sourcing on improving supply chain agility: Global supply chain perspective. International Journal of Production Economics, 187(s/n), 42-52. https://doi.org/10.1016/i. ijpe.2017.02.007

Klein, L. L., \& Pereira, B. A. D. (2016). The survival of interorganizational networks: a proposal based on resource dependence theory. RAM. Revista de Administração Mackenzie, 17(4), 153-175. https:// doi.org/10.1590/1678-69712016/administracao. v17n4p153-175

Kohler, T. (2016). Corporate accelerators: Building bridges between corporations and startups. Business Horizons, 59(3), 347-357. https://doi.org/10.1016/i. bushor.2016.01.008

Kremer, H., Villamor, I., \& Aguinis, H. (2019). Innovation leadership: Best-practice recommendations for promoting employee creativity, voice, and knowledge sharing. Business Horizons, 62(1), 65-74. https://doi.org/10.1016/i. bushor.2018.08.010

Laursen, K., \& Salter, A. (2006). Open for innovation: the role of openness in explaining innovation performance among UK manufacturing firms. Strategic Management Journal, 27(2), 131-150. https://doi.org/10.1002/ smi. 507

Lee, S., Park, G., Yoon, B., \& Park, J. (2010). Open innovation in SMEs-An intermediated network model. Research Policy, 39(2), 290-300. https://doi. org/10.1016/j.respol.2009.12.009

Lefebvre, V. M., Molnár, A., Kühne, B., \& Gellynck, X. (2013). Network competence and open innovation behaviour in the food sector: an empirical investigation. Proceedings in Food System Dynamics, n. esp, 127-149. https://doi.org/10.18461/pfsd.2013.1309

Lin, H. F. (2007). Knowledge sharing and firm innovation capability: An empirical study. International Journal of Manpower, 28(3/4), 315-332. https://doi. org/10.1108/01437720710755272

Mahama, H., \& Cheng, M. M. (2013). The effect of managers' enabling perceptions on costing system use, psychological empowerment, and task performance. Behavioral Research in Accounting, 25(1), 89-114. https://doi.org/10.2308/bria-50333
Mitrega, M., Forkmann, S., Zaefarian, G., \& Henneberg, S. C. (2017). Networking capability in supplier relationships and its impact on product innovation and firm performance. International Journal of Operations \& Production Management, 37(5), 577-606. https://doi. org/10.1108/IJOPM-1 1-2014-0517

Morrissey, W. J., \& Pittaway, L. (2006). Buyersupplier relationships in small firms: the use of social factors to manage relationships. International Small Business Journal, 24(3), 272-298. https://doi. org/10.1177/0266242606063433

O'dell, C., \& Grayson, C. J. (1998). If only we knew what we know: Identification and transfer of internal best practices. California Management Review, 40(3), 154 174. https://doi.org/10.2307/41165948

Parida, V., \& Örtqvist, D. (2015). Interactive effects of network capability, ICT capability, and financial slack on Technology-Based small firm innovation performance. Journal of Small Business Management, 53(1), 278-298. https://doi.org/10.1111/jsbm. 12191

Parida, V., Pesämaa, O., Wincent, J., \& Westerberg, M. (2017). Network capability, innovativeness, and performance: a multidimensional extension for entrepreneurship. Entrepreneurship \& Regional Development, 29(1-2), 94-115. https://doi. org/10.1016/i.jbusres.2015.12.017

Partanen, J., Kohtamäki, M., Patel, P. C., \& Parida, V. (2020). Supply chain ambidexterity and manufacturing SME performance: the moderating roles of network capability and strategic information flow. International Journal of Production Economics, $221(\mathrm{~s} / \mathrm{n}), 107470$. https://doi.org/10.1016/i.ijpe.2019.08.005

Pfeffer, J., \& Salancik, G. (1978). The external control of organizations. New York: Harper and Row.

Podsakoff, P. M., MacKenzie, S. B., Lee, J. Y., \& Podsakoff, N. P. (2003). Common method biases in behavioral research: a critical review of the literature and recommended remedies. Journal of Applied Psychology, 88(5), 879-903. https://doi.org/10.1177/014920638601200408

Popa, S., Soto-Acosta, P., \& Martinez-Conesa, I. (2017). Antecedents, moderators, and outcomes of innovation climate and open innovation: An empirical study in SMEs. Technological Forecasting and Social Change, $118(\mathrm{~s} / \mathrm{n})$, 134-142. https://doi.org/10.1016/i. techfore.2017.02.014

Porter, M. E. (1990). The competitive advantage of nations. Competitive Intelligence Review, 1(1), 1-14.

Radziwon, A., \& Bogers, M. (2019). Open innovation in SMEs: exploring inter-organizational relationships in an ecosystem. Technological Forecasting and Social Change, 146(s/n), 573-587. https://doi.org/10.1016/i. techfore.2018.04.021 
Rakthai, T., Aujirapongpan, S., \& Suanpong, K. (2019). Innovative capacity and the performance of businesses incubated in university incubator units: empirical study from universities in Thailand. Journal of Open Innovation. Technology, Market, and Complexity, 5(2), 1-33. https:// doi.org/10.3390/joitmc5020033

Ries, E. (2011). The lean startup: How today's entrepreneurs use continuous innovation to create radically successful businesses. New York: Random House LLC.

Ritter, T., \& Gemünden, H. G. (2003). Network competence: Its impact on innovation success and its antecedents. Journal of Business Research, 56(9), 745-755. https://doi. org/10.1016/S0148-2963(01)00259-4

Sá, C., \& Lee, H. (2012). Science, business, and innovation: understanding networks in technology-based incubators. R\&D Management, 42(3), 243-253. https:// doi.org/10.1111/j.1467-9310.2012.00681.x

Singh, S. K., Gupta, S., Busso, D., \& Kamboj, S. (2019). Top management knowledge value, knowledge sharing practices, open innovation and organizational performance. Journal of Business Research, n. esp. https:// doi.org/10.1016/j.jbusres.2019.04.040

Spender, J. C., Corvello, V., Grimaldi, M., \& Rippa, P. (2017). Startups and open innovation: a review of the literature. European Journal of Innovation Management, 20(1), 4-30. https://doi.org/10.1108/EJIM-12-2015-0131

Stal, E., Nohara, J. J., \& Chagas, M. F. Jr. (2014). Os conceitos da inovação aberta e o desempenho de empresas brasileiras inovadoras. RAl. Revista de Administração e Inovação, 11 (2), 295-320. https://doi.org/10.5773/rai. v1 1 i2. 1352

Tan, K. H., Wong, W. P., \& Chung, L. (2016). Information and knowledge leakage in supply chain. Information Systems Frontiers, 18(3), 621-638. https://doi. org/10.1007/s10796-015-9553-6

Tehseen, S., \& Sajilan, S. (2016). Network competence based on resource-based view and resource dependence theory. International Journal of Trade and Global Markets, 9(1), 60-82. https://doi.org/10.1504/ IJTGM.2016.074138

Trkman, P., \& Desouza, K. C. (2012). Knowledge risks in organizational networks: An exploratory framework. The Journal of Strategic Information Systems, 21(1), 1-17. https://doi.org/10.1016/i.jsis.2011.11.001

Urbaniec, M., \& Żur, A. (2020). Business model innovation in corporate entrepreneurship: exploratory insights from corporate accelerators. International Entrepreneurship and Management Journal, s/n, 1-24. https://doi. org/10.1007/s 1 1365-020-00646-1

Usman, M., \& Vanhaverbeke, W. (2017). How start-ups successfully organize and manage open innovation with large companies. European Journal of Innovation Management, 20(1), 171-186. https://doi. org/10.1108/EJIM-07-2016-0066

Walter, A., Aver, M., \& Ritter, T. (2006). The impact of network capabilities and entrepreneurial orientation on university spin-off performance. Journal of Business Venturing, 21(4), 541-567. https://doi.org/10.1016/i. jbusvent.2005.02.005

Wang, C., \& Hu, Q. (2020). Knowledge sharing in supply chain networks: Effects of collaborative innovation activities and capability on innovation performance. Technovation, 94, 102010. https://doi.org/10.1016/i. technovation.2017.12.002

Wu, S., Ding, X., Liu, R., \& Gao, H. (2019). How does IT capability affect open innovation performance? The mediating effect of absorptive capacity. European Journal of Innovation Management, 24(1), 43-65. https://doi. org/10.1108/EJIM-02-2019-0043

Yuan, N. (2019). How does the network capability of focal firm affect cluster collaborative innovation? A mediated dual moderation effect model. Management Review, 31 (12), 85-99. 


\section{Apêndice A. Instrumento de pesquisa}

Capacidade de Rede (Partanen et al., 2020; com base em Walter et al., 2006).

$\mathrm{CP}=$ Conhecimento do parceiro; $\mathrm{HR}=$ Habilidades de relacionamento; $\mathrm{CE}=$ Coordenação entre empresas; $\mathrm{Cl}=$ Comunicação interna.

* Item excluído para ajuste do modelo.

Indique até que ponto as afirmações abaixo se aplicam à sua organização em relação à forma, cuidado e uso dos relacionamentos com os seus principais parceiros de inovação aberta (grandes empresas que selecionaram a sua startup para desenvolvimento de inovações). Escala: 1 = não se aplica a 7 = se aplica completamente.

CP1. Em nossa empresa, conhecemos os mercados de nossos parceiros.

CP2. Em nossa empresa, conhecemos os produtos/ procedimentos/serviços de nossos parceiros.

CP3. Em nossa empresa, conhecemos os pontos fortes e fracos de nossos parceiros.

HR1. Em nossa empresa, temos a capacidade de construir boas relações pessoais com nossos parceiros de negócios. HR2. Em nossa empresa, podemos lidar com flexibilidade com nossos parceiros.

HR3. Em nossa empresa, quase sempre resolvemos problemas de forma construtiva com nossos parceiros.

CE1. Em nossa empresa, analisamos o que gostaríamos e desejamos alcançar com qual parceiro.

CE2. Em nossa empresa, desenvolvemos relações com cada parceiro com base no que eles podem contribuir.

CE3. Em nossa empresa, discutimos regularmente com nossos parceiros como podemos nos apoiar.

Cl1. Em nossa empresa, temos reuniões regulares para todos os projetos.

Cl2. Em nossa empresa, os funcionários desenvolvem contatos informais entre si.*

CI3. Em nossa empresa, gerentes e funcionários geralmente dão feedback um ao outro.

Compartilhamento de Informações (Cl) (Cheng, 2011; Tan et al., 2016).

Indique seu grau de concordância com cada uma das assertivas abaixo sobre compartilhamento de informações no âmbito do relacionamento da sua organização com os seus principais parceiros de inovação aberta (grandes empresas que selecionaram a sua startup para desenvolvimento de inovações). Escala: 1 = discordo totalmente a 7 = concordo totalmente.
Cl1. Nossos parceiros compartilham informações proprietárias conosco.

Cl2. Fornecemos informações ao nosso parceiro que podem ajudá-lo.

Cl3. Fornecemos informações ao nosso parceiro de forma frequente e informal, e não apenas de acordo com o contrato específico.

Cl4. Nós e nossos parceiros trocamos informações que ajudam no planejamento dos negócios.

Compartilhamento de Conhecimento (CC) (Wang \& Hu, 2020).

Indique seu grau de concordância com cada uma das assertivas abaixo sobre compartilhamento de conhecimento no âmbito do relacionamento da sua organização com os seus principais parceiros de inovação aberta (grandes empresas que selecionaram a sua startup para desenvolvimento de inovações). Escala: 1 = discordo totalmente a 7 = concordo totalmente

CC1. Compartilhamos nossos relatórios de trabalho de inovação e documentos técnicos com nossos parceiros, mediante solicitação.

CC2. Compartilhamos nossos manuais e metodologias com nossos parceiros, a pedido deles.

CC3. Frequentemente compartilhamos nossa experiência, know-how ou novas ideias com nossos parceiros.

Inovação Aberta (IA) (Hameed et al., 2018).

Indique seu grau de concordância com cada uma das assertivas abaixo relativas ao desempenho da inovação aberta (grandes empresas que selecionaram a sua startup para desenvolvimento de inovações). Escala: $1=$ discordo totalmente a 7 = concordo totalmente.

IA1. Optamos por nos engajar no modelo de inovação aberta, acreditando que é uma forma de comercializar a ideia.

IA2. Os esforços de colaboração com parceiros fora da organização para trabalhar em um projeto de ganho mútuo são a melhor descrição de inovação aberta.

IA3.Optamos por nos engajar no modelo de inovação aberta, acreditando que a terceirização de expertise é benéfica para nossa organização.

IA4. Novas ideias são sempre bem-vindas para a inovação aberta em nossa organização.

IA5. O licenciamento out-or-in de propriedade intelectual é a melhor descrição de inovação aberta.

IA6. O compartilhamento de conhecimento interno e externo aumenta a inovação aberta.

IA7. O licenciamento das ideias mais recentes promove a inovação aberta. 